\title{
In Vitro Effects of Tire Debris Organic Extracts on Human Spermatozoa
}

\author{
Michela Geminiani ${ }^{1}$, Elena Moretti ${ }^{1,2}$, Francesco Cetta $^{3}$, Cesare Castellini $^{4}$, Gaia Terzuoli ${ }^{1}$, \\ Lucia Mazzi $^{1}$ and Giulia Collodel*,1,2
}

\author{
${ }^{1}$ Department of Biomedical Sciences, Applied Biology Section; University of Siena; ${ }^{2}$ Interdepartmental Centre for \\ Research and Therapy of Male Infertility, University of Siena; ${ }^{3}$ Department of Surgery, University of Siena; \\ ${ }^{4}$ Department of Applied Biology, Animal Science Section, University of Perugia
}

\begin{abstract}
Background \& objectives: Environmental pollutants have detrimental effects on the reproductive function of human being and of animals. This study was aimed at evaluating the effects of tire debris, a fraction of particulate matter derived from tire wear, on motility and morphology of human spermatozoa.

Methods: spermatozoa from ten healthy men were incubated for 4 hours with 10, 50 and $75 \mu \mathrm{g} / \mathrm{ml}$ of tire debris organic extract (TDOE). Sperm motility and morphology were evaluated according to WHO guidelines; the presence of apoptosis and necrosis was assessed by the AnnexinV/Propidium iodide assay. Ultrastructure of sperm organelles was observed by transmission electron microscopy.

Results: incubation with $10 \mu \mathrm{g} / \mathrm{ml}$ of TDOE did not affect sperm quality, except for the value of apoptosis (median: 8\% vs. $6 \% ; \mathrm{P}<0.01)$. Sperm motility and sperm morphology percentages $(\mathrm{P}<0.01)$ decreased when sperm were treated with 50 and $75 \mu \mathrm{g} / \mathrm{ml}$ TDOE and the percentage of apoptotic and necrotic sperm $(\mathrm{P}<0.01)$ increased concomitantly. At the same concentrations TEM analysis showed an increased percentage of reacted and swollen acrosomes, disrupted chromatin and altered axonemes.

Interpretation \& conclusions: the organic part of tire debris is potentially harmful to spermatozoa, particularly at a concentration of 50-75 $\mu \mathrm{g} / \mathrm{ml}$, inhibiting motility and compromising morphological integrity. Nevertheless, these results cannot yet be extrapolated to the in vivo outcome due to the lack of studies focused on this issue.
\end{abstract}

Keywords: Human semen quality, particulate matter, tire debris organic extracts, TEM.

\section{INTRODUCTION}

Tire debris (TD) are produced by the wear of tires from motor vehicles and they have been found suspended in the urban atmosphere and deposited along the roadside [1]; the literature [2] has shown that these particles are $5-7 \%$ of the particulate matter breathable fraction $\left(\mathrm{PM}_{10}\right)$. In general, $\mathrm{PM}$ is a complex mixture of dust, dirt and soot or smoke and liquid droplets suspended in the air. PM standards are based on total mass and size, which can range from a few nanometers to tens of micrometers in an aerodynamic diameter $(2.5 \mu \mathrm{m}$ to $10 \mu \mathrm{m})$ [3]. Exposure to breathable PM has been associated with an exacerbation of asthma and chronic obstructive pulmonary disease, as well as with increased morbidity due to respiratory and cardiovascular diseases [4]. The mechanisms underlying PM toxicity are not fully known; inflammation, oxidative stress [5] and DNA damages [6] seem to be involved.

Environmental exposure to PM leads to a possibility that TD can be inhaled by inhabitants, and the toxicity of this component should be considered. TD has only recently received attention as a component of PM and its chemical

*Address correspondence to this author at the Department of Biomedical Sciences, Applied Biology Section, University of Siena, Policlinico Le Scotte, Viale Bracci, 14, 53100 Siena, Italy; Tel: 39-0577-233539;

Fax: 39-0577-233527; E-mail: collodel@unisi.it content has been analyzed. TD toxicity has been linked to inorganic components such as zinc [7] and to organic compounds [8].

Although the potential toxicity of zinc leached by tire material on water biota has been extensively described, the toxicity derived from the presence of organic compounds has received little attention. The organic portion of TD is mainly characterized by the presence of isoprene polymers and other organic hydrocarbons, such as long chain alkanes [9], and it has been demonstrated to induce a dose-dependent increase in cell mortality, DNA damage and a significant modification in cell morphology [10]. For example, Gualtieri et al. [11] tested the effects of TD organic extracts at 10,50, 60 and $75 \mu \mathrm{g} / \mathrm{ml}$ concentrations on the human lung A549 cell line for 24, 48 and 72 hours; the concentrations were calculated by considering the amount of TD inhaled daily by humans from the air of a polluted urban area. This research demonstrated that TD extract, particularly at a concentration of $60 \mu \mathrm{g} / \mathrm{ml}$, induced a dose dependent increase in cell mortality and DNA damage, concomitant with severe ultrastructural alterations.

The decline in semen quality, a phenomenon that occurs in developed countries, has been suggested to be associated with enhanced exposure to environmental chemicals that act as endocrine disrupters as a result of increased use of pesticides, plastics, and other anthropogenic materials. A 
significant amount of toxicology data based upon laboratory and wildlife animals studies has suggested that exposure to certain chemicals is associated with reproductive toxicity involving reduced semen quality parameters (sperm concentration, motility, morphology) and impaired fertility [12].

Semen could be considered the more accessible reproductive endpoint, which can be easily analyzed by well established procedures [13]. Basic semen analysis measures sperm concentration, viability, motility and morphology, however other assays could be required in order to better understand the mechanisms underlying adverse chemical effects on spermatozoa.

Because TD are a consistent component in an urban atmosphere and inhabitants are exposed to this kind of pollutant, we hypothesized that TD may influence reproductive function.

The present study was aimed at investigating whether organic TD extract (TDOE) may have an adverse in vitro effect on human spermatozoa.

\section{MATERIALS AND METHODOLOGY}

\section{Tire Particles and Organic Extracts}

Tire debris $(<100 \mu \mathrm{m})$ were produced in laboratory wear test with pieces of new vehicle wheel milled in liquid nitrogen. We obtained these particles from Prof. E. Bolzacchini (Department of Environmental Sciences, University of Milano-Bicocca). The TDOE was prepared according to the method proposed by Gualtieri et al. $[14,15]$ and by means of a Soxhlet extraction apparatus (SER 148, VELP ${ }^{\circledR}$ SCIENTIFICA, Milan, Italy). TD were Soxhletextracted for 6 hours with $150 \mathrm{ml}$ of dichloromethane and then dried under a flow of nitrogen. In order to render the organic fractions soluble, TDOE were suspended in sterile dimetylsulfoxide (DMSO 0.5\%, Sigma-Aldrich, Italy), in which no obvious toxicity to spermatozoa was observed, at a final concentration of $10 \mathrm{mg} / \mathrm{ml}$ and stored at $-20{ }^{\circ} \mathrm{C}$.

\section{Human Semen Samples}

Semen samples were furnished by 10 healthy men (aged 25-35) with normal semen parameters referred to the Interdepartmental Centre for Research and Therapy of Male Infertility, Siena University for semen analysis. The criteria for participation in the study included the presence of a normal karyotype and the absence of anatomical problems, such as varicocele or cryptorchidism, or genitourinary infections. All of the subjects that participated in the study gave informed consent for this research.

Samples were collected by masturbation after 4 days of sexual abstinence and examined after complete liquefaction for $30 \mathrm{~min}$ at $37{ }^{\circ} \mathrm{C}$. Volume, $\mathrm{pH}$, concentration, motility were evaluated according to World Health Organization guidelines [13] and morphology was examined by Papanicolau staining modified for spermatozoa. Ejaculated human spermatozoa were washed twice in phosphate buffered saline (PBS), smeared on pre-cleaned glass slides, air dried and fixed in equal volume of ethanol $95 \%$ and ether, for 5-15 min. The fixed slides were stained and evaluated using the protocol reported in WHO guidelines [16].
Human ejaculated spermatozoa $\left(10 \times 10^{6}\right.$ cells $)$ were incubated with TDOE in Biggers, Whitten, Whittingham (BWW) buffer at a concentration of 10,50 and $75 \mu \mathrm{g} / \mathrm{ml}$ for 4 hours at $37{ }^{\circ} \mathrm{C}$ with $5 \% \mathrm{CO}_{2}$. The range of concentrations was selected considering the data reported Gualtieri et al. [11]. Aliquots of samples not incubated with TDOE were used as controls.

\section{Electron Microscopy}

Ultrastructural sperm analysis was carried out in the considered samples by transmission electron microscopy (TEM). For TEM, sperm samples were fixed in cold Karnovsky fixative and maintained at $4^{\circ} \mathrm{C}$ for 2 hours. Fixed semen was washed in $0.1 \mathrm{~mol} / 1$ cacodylate buffer $(\mathrm{pH} 7.2)$ for 12 hours, postfixed in $1 \%$ buffered osmium tetroxide for 1hour at $4^{\circ} \mathrm{C}$, then dehydrated and embedded in Epon Araldite (Sigma-Aldrich, Italy). Ultra-thin sections were cut with a Supernova ultramicrotome (Reickert Jung, Vienna, Austria), mounted on copper grids, stained with uranyl acetate and lead citrate and then observed and photographed with a EM208 transmission electron microscope (TEM; Philips Scientifics, Eindhoven, The Netherlands).

A minimum of 300 sperm sections were analyzed for each sample and the anomalies related to the acrosome (reacted, swollen), the chromatin (disrupted), the axoneme (disorganized) and the plasma membrane (broken) were quantified.

\section{Annexin V/Propidium Iodide Assay}

AnnexinV/Propidium iodide assay was carried out in treated and untreated human samples to quantify the presence of necrosis and apoptosis in all analysed sperm samples. A critical stage of apoptosis involves the acquisition of surface changes, in particular phosphatidylserine (PS) is translocated from the inner side of the plasma membrane to the outer layer. The Annexin V protein preferentially binds to negatively charged phospholipids, such as PS in the presence of $\mathrm{Ca}^{2+}$, and it shows minimal binding to phosphatidilcholine and sphingomyeline. Propidium iodide (PI) penetrates cells with broken membranes, considered necrotic cells. The detection of PS externalization was performed with the Vybrant Apoptosis Assay kit (Invitrogen Ldt, UK) made up of Annexin V-FITC and PI that are able to differentiate viable from necrotic cells. Samples were washed with phosphate buffered saline (PBS), centrifuged and suspended in Annexin-binding buffer (ABB) to obtain a cell density of $1 \times 10^{6}$. Following the manufacturer's instructions, $10 \mu \mathrm{l}$ of conjugated-FITC Annexin V and $1 \mu \mathrm{l}$ of PI $(100 \mu \mathrm{g} / \mathrm{ml})$ working solution were added to each $100 \mu \mathrm{l}$ of cell suspension. The spermatozoa were incubated at room temperature for $15 \mathrm{~min}$. After a careful wash with $\mathrm{ABB}$, a drop of sperm cell suspension was smeared on each glass slide. Slides were mounted in glycerol containing 5\% npropylgallate. Observations were made and photographs were taken with a Leitz Aristoplan (Leica, Wetzlar, Germany) light microscope equipped with a fluorescence apparatus. A total of 100 spermatozoa from each sample were scored. By staining cells with FITC-Annexin V (AnV, green fluorescence), and simultaneously with the non vital dye Propidium iodide (PI, red fluorescence), it is possible to recognize intact cells ( $\mathrm{AnV}$ negative, PI negative), early 
apoptotic cells (AnV positive, PI negative), damaged sperm with PS externalization (AnV positive, PI positive) and damaged necrotic sperm (AnV negative, PI positive).

\section{Statistical Analysis}

Statistical analysis was performed using the version 8 SAS system (Sas Institute Inc. Cary, NC 27513, USA). The Kolmogorov-Smirnov test was used to verify normal or nonnormal distribution of values. The significance of the differences within the characteristics of basal samples and treated samples was evaluated with the Kruskal-Wallis test since the variables were not normally distributed. When a statistically significant difference was found among the groups, the Mann- Whitney U-Post hoc test was then used between pairs of groups. $\mathrm{P}<0.05$ was considered to be significant.

\section{RESULTS}

Ten human semen samples were examined by optical microscopy and classified as normal according to WHO guidelines $[13,16]$ : the median and range were respectively reported in parenthesis for concentration, measured as sperm $/ \mathrm{ml}\left(45 \times 10^{6}, 20-64 \times 10^{6}\right)$, motility percentage $(55,50-$ $63)$ and normal morphology percentage (32, 30-37).

Aliquots of all analysed specimens were incubated for 4 hours with 10,50 and $75 \mu \mathrm{g} / \mathrm{ml}$ TDOE and untreated semen under the same conditions was used as a control.

Sperm motility and morphology were evaluated in all treated and untreated specimens, which were then processed by Annexin V/PI assay. The values obtained for each considered characteristic in each examined TDOE incubation are reported in Table $\mathbf{I}$.

The results of these experiments demonstrated that the incubation of human semen with $10 \mu \mathrm{g} / \mathrm{ml}$ of TDOE for 4 hours did not affect semen quality. The only significantly different value between untreated semen and semen incubated with $10 \mu \mathrm{g} / \mathrm{ml}$ of TDOE was phosphatidylserine externalization $(\mathrm{An}+\mathrm{PI}-; \mathrm{P}<0.01)$, reputed to be one of the first steps of the apoptotic process. The situation appeared dramatically different when the concentration of TDOE was increased $(\geq 50 \mu \mathrm{g} / \mathrm{ml})$. In these cases, the sperm motility, the sperm morphology, the intact sperm, evaluated by Annexin V/PI assay, significantly decreased $(\mathrm{P}<0.01)$ versus controls or $10 \mu \mathrm{g} / \mathrm{ml}$ TDOE treated sperm. The same significant decrement was observed when the same variables were compared between sperm treated with $50 \mu \mathrm{g} / \mathrm{ml}$ and those treated with $75 \mu \mathrm{g} / \mathrm{ml}$ (Table I). The progressive decline in these sperm parameters by increasing TDOE concentration was concomitant with a significant increase in the percentage of sperm with PS externalization (apoptosis), with broken plasma membrane (necrosis) and with both phenomena $(\mathrm{P}<0.01)$ (Table I).

In the same samples, ultrastructural characteristics of sperm organelles were evaluated. The percentages of the most frequent alterations of the acrosome, the chromatin packaging, the axoneme and the plasma membrane strongly increased in samples treated with 50 and $75 \mu \mathrm{g} / \mathrm{ml}$ of TDOE compared to $10 \mu \mathrm{g} / \mathrm{ml}$ and controls (Fig. 1). In Figs (2) and (3) examples of sperm treated with 10 and $50 \mu \mathrm{g} / \mathrm{ml}$ were shown.

Table I. Characteristic Examined in Sperm After TDOE Incubation

In Vitro Effects of TDOE at Different Concentrations After 4 Hours of Exposure on Motility (\%), Apoptosis and Necrosis (\%) and Morphology of Spermatozoa of 10 Individuals with Normal Semen Parameters. Medians and Ranges (Lower and upper Limits) of Analyzed Characteristics are Shown. The Significant Values Reported in the Last Column were Obtained by applying the Mann Whitney U- Post Hoc Test Among the Pairs of Groups

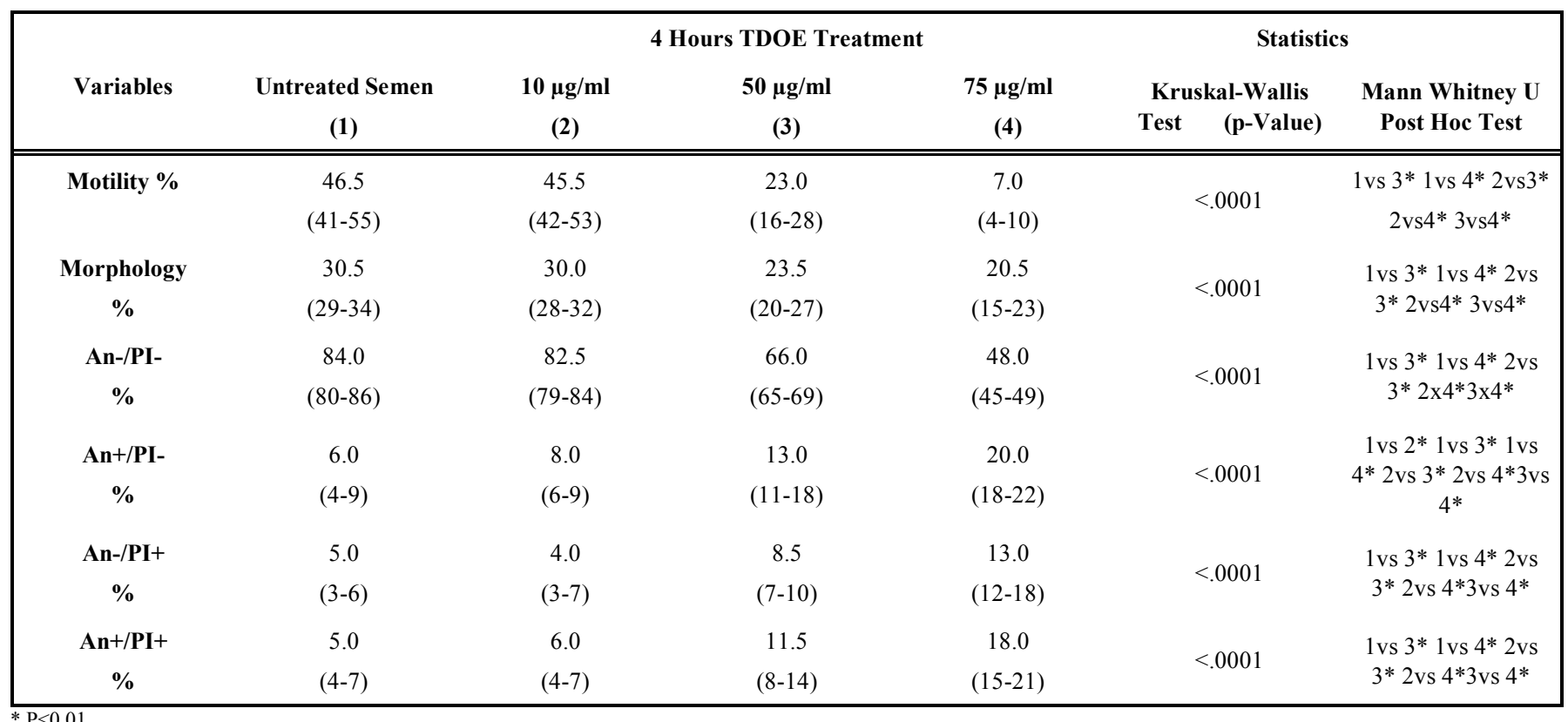

Legend: Motility \% (percentage of rapid + slow progressive motility); Morphology \% (percentage of normal spermatozoa evaluated by Papanicolaou staining modified for spermatozoa), AnV- PI- (percentage of intact sperm evaluated with Annexin V/Propidium Iodide assay), AnV + PI - (percentage of sperm with PS externalization indicating apoptosis), AnV- PI + (percentage of sperm with fragmented plasma membrane indicating necrosis), AnV+ PI + (percentage of sperm with PS externalization and broken plasma membrane). 


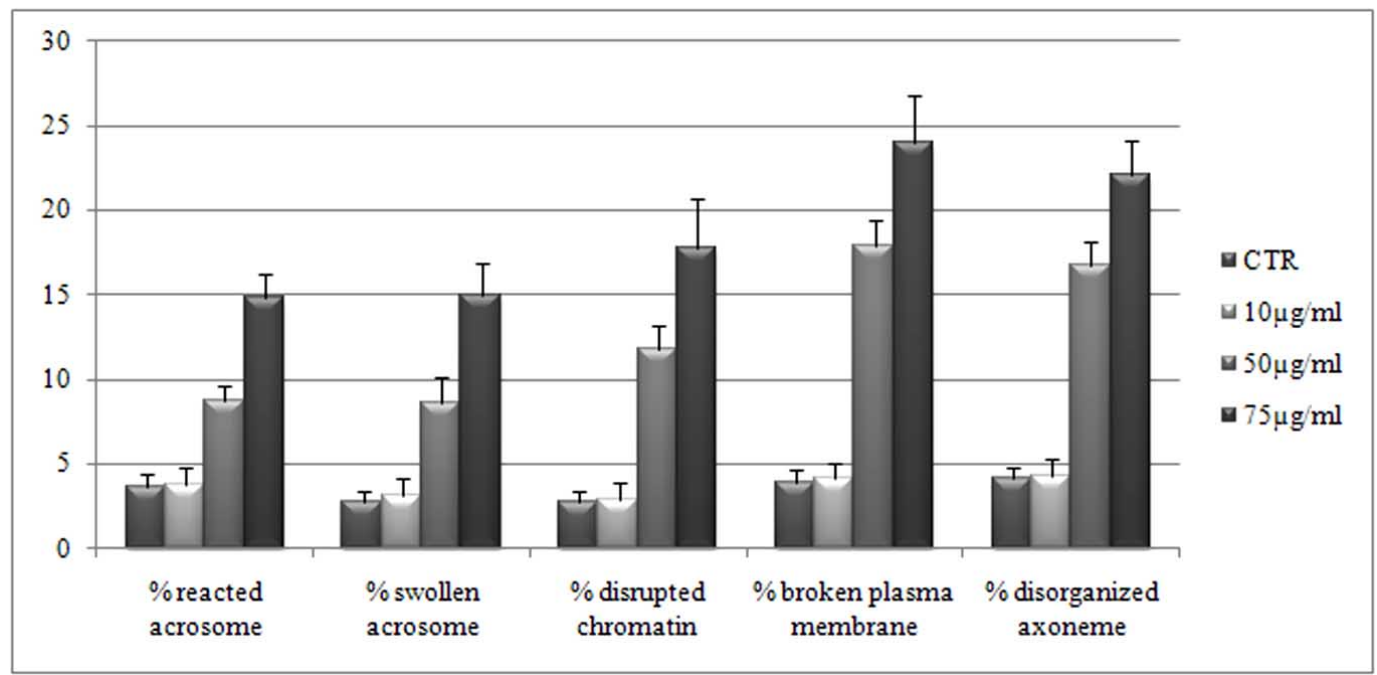

Fig. (1). Mean and standard deviation in the percentage of the most frequent alterations (acrosome, chromatin, axoneme, plasma membrane) observed by TEM in human sperm treated with $75 \mu \mathrm{g} / \mathrm{ml}$ and controls.

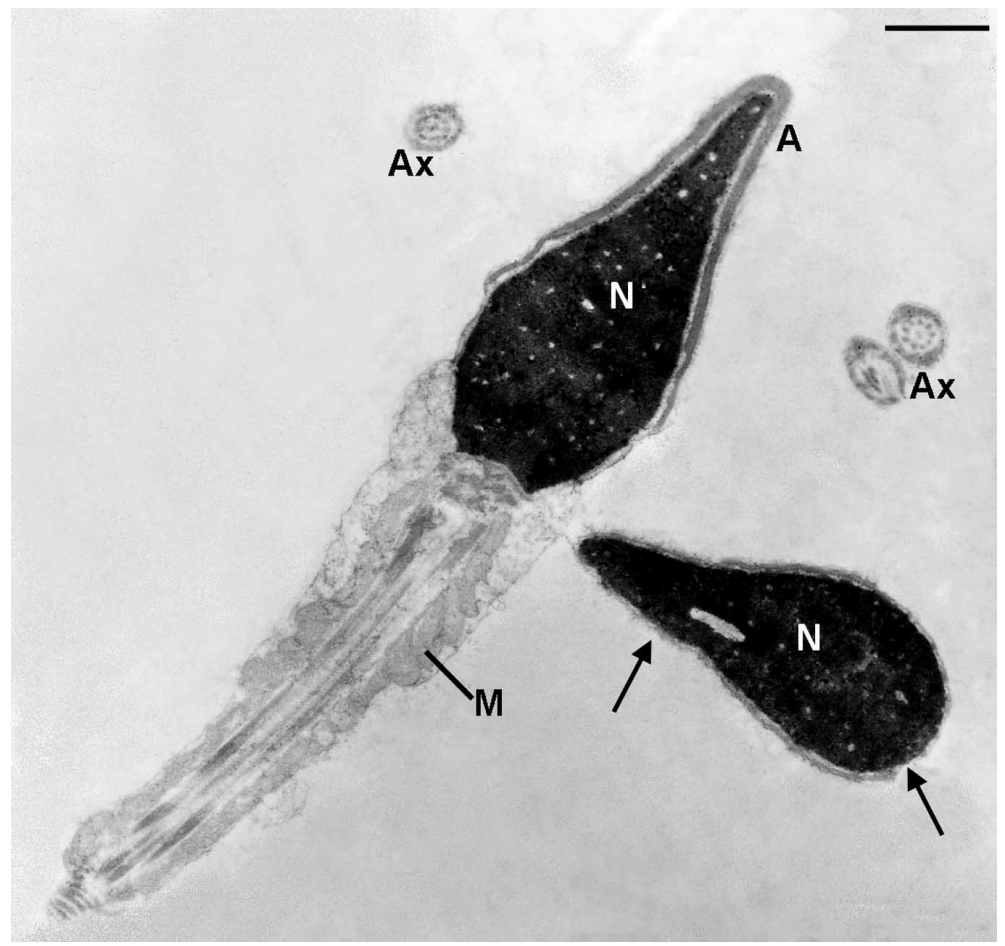

Fig. (2). TEM micrograph of longitudinal sections of human spermatozoa after 4 hours of incubation with $10 \mu \mathrm{g} / \mathrm{ml}$ TDOE. The left spermatozoon shows a normal acrosome $(\mathrm{A})$ and nucleus $(\mathrm{N})$ although the mitochondria are slightly swollen (M). The right spermatozoon shows a well shaped nucleus $(\mathrm{N})$ without an acrosome and a broken plasma membrane (arrows). Axoneme (Ax). Bar $1 \mu \mathrm{m}$.

\section{DISCUSSION}

Environmental pollutants have detrimental effects on the reproductive function of human beings and of animals. Tire debris, a fraction of $\mathrm{PM}_{10}$, have recently received enough attention that it can be asserted that they have an impact on living organisms in general [14] and on human cell lines $[8,11,15]$.

The present study sheds a light on the impact of TDOE exposure on human sperm using an in vitro cellular model. To our knowledge, this is the first study that has addressed the issue of evaluating the morphology of human spermatozoa after exposure to organic TD extract by means of fluorescent assay and TEM analysis.

The concentrations to be used were selected on the basis of experimental evidence from studies performed on other cellular models $[9,11]$. In particular, Gualtieri et al. [11] tested the effects of TDOE at 10,50,60 and $75 \mu \mathrm{g} / \mathrm{ml}$ concentrations on the human lung A549 cell line for 24, 48 and 72 hours. In this paper it was reported that the concentrations were calculated by considering the amount of TD in the air, of a polluted urban area, inhaled daily by humans. The time of exposure was extrapolated from an in vitro rabbit sperm model for metal compound and TDOE 


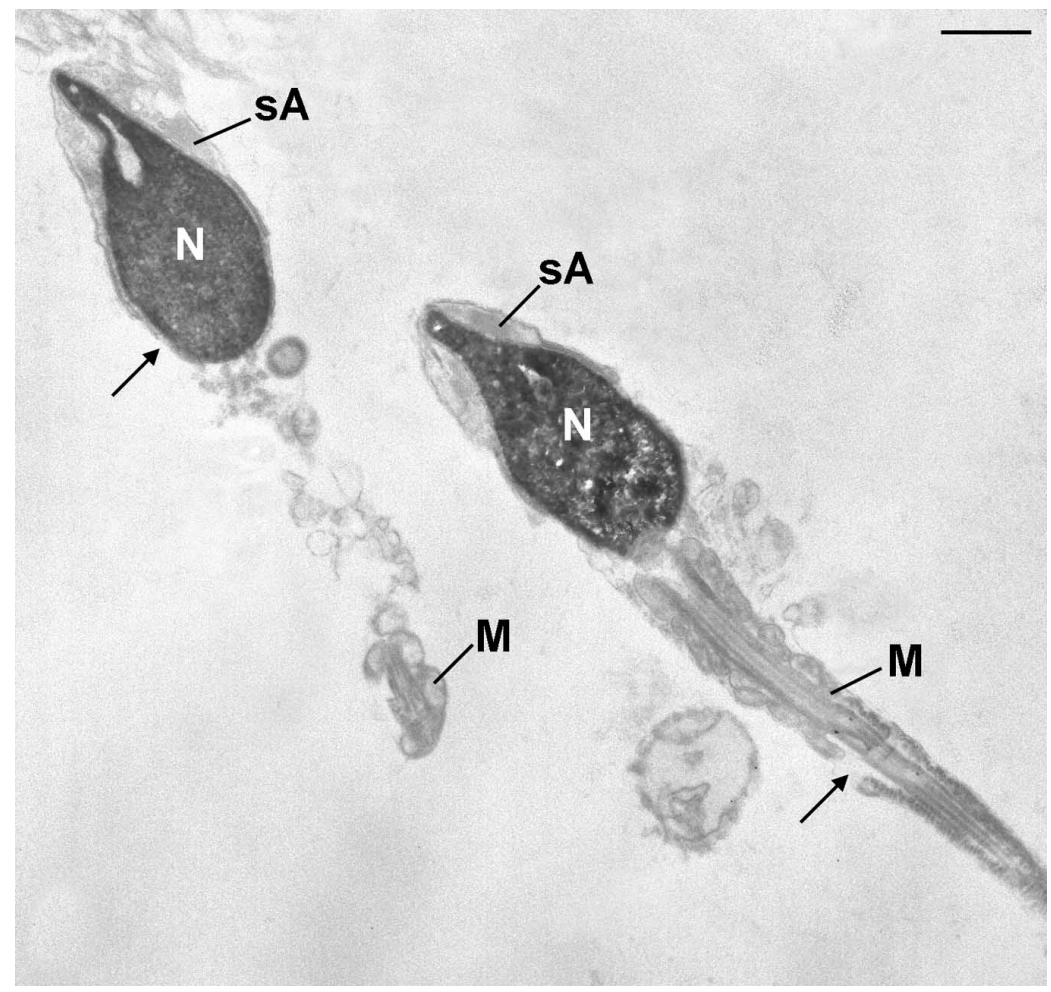

Fig. (3). TEM micrograph of longitudinal sections of human spermatozoa after 4 hours of incubation with $50 \mu \mathrm{g} / \mathrm{ml}$ TDOE. Acrosomes are swollen (sA) and plasma membranes are broken (arrows). Mitochondria (M) are altered and disassembled. Nucleus (N). Bar $1 \mu \mathrm{m}$.

toxicity $[17,18]$; considering that spermatozoa are highly differentiated and partially inactive cells from the transcriptional point of view, the incubation times are different from those used for other cells.

Regarding motility, a significant dose-response relationship was observed after 50 and $75 \mu \mathrm{g} / \mathrm{ml}$ TDOE treatment. It is well known that increased reactive oxygen species (ROS) production is negatively associated with human sperm motility [19]; for this reason, we might hypothesize that ROS induced by an organic compound of tire particles could act as a motility depressor. On the other hand, the involvement of TDOE in inducing ROS and heat shock proteins in the A549 human alveolar cell line has recently been demonstrated [10].

The decline in sperm quality caused by exposure to 50 and $75 \mu \mathrm{g} / \mathrm{ml}$ was clearly demonstrated by optical as well as electronic microscopy.

The incubation of human spermatozoa with 50 and 75 $\mu \mathrm{g} / \mathrm{ml}$ of TDOE caused structural sperm defects, particularly at the membrane head level, detected by the significant increase in cells with PS externalization and broken plasma membrane, highlighted by PI treatment. The Papanicolaou staining, used to evaluate the sperm morphology, highlighted mainly altered or absent acrosomes.

TEM analysis of sperm sections confirmed these observations, underlining diffuse plasma membrane breakage associated with injuries to the acrosome, that were often absent or reacted. Concomitantly the high resolution power of TEM allowed to observe alterations of the chromatin and axonemes.
Damages at the plasma membrane level have also been reported in human lung epithelial cells after in vitro TDOE treatment [9]. Regarding spermatozoa, the higher vulnerability of the plasma membrane region coating the acrosome cap is probably due to its physiological role; this organelle is characterized by a certain instability to ensure a natural break down after interaction with the zona pellucida of an oocyte. Nevertheless, molecular studies should be performed to better elucidate this aspect. In addition, structural difference between the membranes of different cells such as sperm and epithelial lung cells cannot be excluded.

Beyond plasma membrane breakage, another important structural change in spermatozoa occurred after the TDOE challenge: the translocation of PS from the inner to the outer leaflet of the plasma membrane, observed even at TDOE 10 $\mu \mathrm{g} / \mathrm{ml}$ treatment.

This modification in lipid architecture has been considered as one of the earliest signs of apoptosis, although it has also been suggested that PS externalization in human spermatozoa can be related to the acrosome reaction rather than to apoptosis, mainly due to capacitation required before, acrosome reaction [20]. Taking these considerations into account, it can be hypothesized that TDOE may induce a precocious acrosome reaction, making sperm unable to fertilize an egg. These hypotheses, merely speculative, deserve other study to be demonstrated.

A question that may naturally arise is how these compounds may reach the reproductive organs in vivo and cause possible damage. The blood-testicular barrier is very selective, protecting these cells from most toxic agents that 
are accidentally introduced into the organism. An intriguing hypothesis is that TD, which consists of organic and inorganic compounds, may accumulate in the white adipose tissue, a known reservoir of lipophilic environmental pollutants [21]. As for other hydrophobic environmental contaminants, TD may alter the metabolism and function of the adipose tissue and be chronically released throughout the whole organism. Furthermore, conditions compromising the integrity of the blood-testicular barrier, such as anatomical pathologies or inflammation/infection of the male genital tract, may render the access of TD to the reproductive organs feasible. Whether TD can accumulate in the adipose tissue and pass through the blood-testicular barrier will have to be established by in vivo studies, possibly using animal models.

In conclusion, our results, although preliminary, lend support to the evidence that the organic part of tire debris is potentially harmful to spermatozoa even at $10 \mu \mathrm{g} / \mathrm{ml}$ and in particular over a threshold level $(50-75 \mu \mathrm{g} / \mathrm{ml})$, inhibiting their motility and compromising their morphological integrity. We are aware that the sample size is small anyway significant differences among the considered variables were found and the parameters of basal samples were enough homogeneous.

In addition, the concentrations tested are quite high and the best way to overcome this problem will be to test the effects of TD and particulate matter in an animal model such as rat.

Nevertheless, these results obviously still cannot be extrapolated to an in vivo outcome due to a lack of studies focused on this problem.

\section{ACKNOWLEDGEMENT}

Supported by the PROLIFE Flagship project, city of Milan, Italy.

\section{REFERENCES}

[1] Fauser P. Particulate air pollution with emphasis on traffic generated aerosols. Riso National Laboratory ISBN 1999; 87-5502532-3.

[2] Tappe M, Null V. Requirements for tires from the environmental view point. In: Tire Technology Expo Conference, Hamburg. 2002

[3] Wegesser TC, Last JA. Lung response to coarse PM: Bioassay in mice. Toxicol Appl Pharmacol 2008; 230: 159-66.
[4] Alexis NE, Lay JC, Zeman K et al. Biological material on inhaled coarse fraction particulate matter activates airway phagocytes in vivo in healthy volunteers. J Allergy Clin Immunol 2006; 117 : 1396-403.

[5] Risom L, Møller P, Loft S. Oxidative stress-induced DNA damage by particulate air pollution. Mutat Res 2005; 592: 119-37.

[6] Sørensen M, Autrup H, Hertel O, Wallin H, Knudsen LE, Loft S. Personal exposure to PM2.5 and biomarkers of DNA damage. Cancer Epidemiol Biomarkers Prev 2003; 12: 191-6.

[7] Smolders E, Degryse F. Fate and effect of zinc from tire debris in soil. Environ Sci Technol 2002; 36: 3706-10.

[8] Camatini M, Calini V, Gualtieri M. Biological and chemical evaluation of tire debris organic extract. Toxicol Lett 2003; 144 : 182 .

[9] Beretta E, Gualtieri M, Botto L, Palestini P, Miserocchi G, Camatini M. Organic extract of tire debris causes localized damage in the plasma membrane of human lung epithelial cells. Toxicol Lett 2007; 173: 191-200.

[10] Gualtieri M, Mantecca P, Cetta F, Camatini M. Organic compounds in tire particle induce reactive oxygen species and heatshock proteins in the human alveolar cell line A549. Environ Int 2008; 34: 437-42.

[11] Gualtieri M, Rigamonti L, Galeotti V, Camatini M. Toxicity of tire debris extracts on human lung cell line A549. In vitro Toxicol 2005; 19: 1001-8.

[12] Phillips KP, Tanphaichitr N. Human exposure to endocrine disrupters and semen quality. J Toxicol Environ Health B Crit Rev 2008; 11: 188-220.

[13] World Health Organization. WHO laboratory manual for the examination of human semen and sperm-cervical mucus interaction. 4th ed. Cambridge: Cambridge University Press 1999.

[14] Gualtieri M, Andrioletti M, Mantecca P, Vismara C, Camatini M. Impact of tire debris on in vitro and in vivo systems. Part Fibre Toxicol 2005; 2: 1 .

[15] Gualtieri M, Andrioletti M, Vismara C, Milani M, Camatini M. Toxicity of tire debris leachates. Environ Int 2005; 31: 723-30.

[16] World Health Organization. WHO laboratory manual for the examination of human semen and semen-cervical mucus interaction. Cambridge, United Kingdom: Cambridge University Press 1992

[17] Castellini C, Mourvaki E, Sartini B et al. In vitro toxic effects of metal compounds on kinetic traits and ultrastructure of rabbit spermatozoa. Reprod Toxicol 2009; 27: 46-54.

[18] Moretti E, Dal Bosco A, Mourvaki E, et al. In vitro effects of the tire debris organic extract on the kinetic and morphologic traits of rabbit spermatozoa WRS 2009; 17: 213-20.

[19] Tremellen K. Oxidative stress and male infertility--a clinical perspective. Hum Reprod Update 2008; 14: 243-58.

[20] Martin G, Sabido O, Durand P, Levy R. Phosphatidylserine externalization in human sperm induced by calcium ionophore A23187: relationship with apoptosis, membrane scrambling and the acrosome reaction. Hum Reprod 2005; 20: 3459-68.

[21] Müllerová D, Kopecký J. White adipose tissue: storage and effector site for environmental pollutants. Physiol Res 2007; 56: 375-81. 